\title{
Heterogeneity and intermediates turnover during amyloid- $\beta(A \beta)$ peptide aggregation studied by Fluorescence Correlation Spectroscopy
}

\author{
Ann Tiiman ${ }^{\dagger}$, Jüri Jarvet ${ }^{\ddagger}$, Astrid Gräslund ${ }^{\dagger}$ and Vladana Vukojevic $^{\perp, *}$ \\ †Department of Biochemistry and Biophysics, Arrhenius Laboratories, Stockholm \\ University, 10691 Stockholm, Sweden \\ $\ddagger$ The National Institute of Chemical Physics and Biophysics, Akadeemia tee 23, 12618 Tallinn, Estonia \\ $\perp$ Department of Clinical Neuroscience, Center for Molecular Medicine CMM L8:01, Karolinska \\ Institutet, 17176 Stockholm, Sweden
}

Matherials and Methods S2

Analysis of autocorrelation curves S4

Effect of FCS measurement time on autocorrelation curves S6

Effect of brightness on FCS analysis S8

Table S1. Kinetic parameters obtained from FCS measurements S10

Figure S1. Analysis of ACCs recorded in a solution of $10 \mu \mathrm{M} A \beta 42$ and $10 \mu \mathrm{M}$ ThT $\ldots$ S11

Figure S2. FCS measurements in a $25 \mathrm{nM}$ standard solution of Rh6G in water S12

Figure S3. FCS measurements in a standard solution of quantum dots in water S13

Figure S4. FCS measurements in a standard solution of quantum dots in $40 \%$ sucrose

Figure S5. FCS measurements in a standard solution of quantum dots in $60 \%$ sucrose

Figure S6. FCS measurements in a standard solution of quantum dots in $70 \%$ sucrose

Figure S7. Effect of different ACC evaluation methods on the overall distribution of diffusion times measured in a solution of $10 \mu \mathrm{M} A \beta 42$ and $10 \mu \mathrm{M}$ ThT.

Figure S8. Fitting of the distribution of diffusion times measured by FCS.

Changes in the number of ThT-reactive aggregates with different diffusion times monitored using $10 \mu \mathrm{M} A \beta$ and different ThT concentrations 


\section{Materials and methods}

Materials Lyophilized A $\beta 42$ (ultra-pure, recombinant) was purchased from rPetide(Bogart, USA). A $\beta 40$ was from AlexoTech AB (Umeå, Sweden). Carboxylate functionalized quantum dot nanocrystals, $d=20 \mathrm{~nm}$, emission maxima at $525 \mathrm{~nm}\left(\mathrm{Qdot}{ }^{\circledR} 525\right.$ ITK $^{\mathrm{TM}}$ Carboxyl Quantum Dots) were purchased from Molecular Probes, Life Technologies Corporation, USA.

Sample preparation A $\beta 42$ was dissolved in hexafluoroisopropanol (HFIP) and dried in a vacuum exicator. No prior treatment was done with $A \beta 40$. A $\beta$ solutions were freshly prepared before each experiment by dissolving the HFIP film/peptide powder in $10 \mathrm{mM}$ $\mathrm{NaOH}$ to a concentration of $1.0 \mathrm{mg} / \mathrm{ml}$ and then diluted to the final concentration using 20 mM HEPES containing $10 \mu \mathrm{M}$ ThT, unless stated otherwise. For CD experiments the sample was diluted to the final concentration with water and the $\mathrm{pH}$ was adjusted with $\mathrm{NaH}_{2} \mathrm{PO}_{4}$, resulting in a $0.5 \mathrm{mM}$ phosphate buffer. The sample preparation was done on ice.

$1 \mathrm{ml}$ of the solution was transferred to a quartz spectroscopic cuvette $(4 \times 10 \mathrm{~mm})$, with a rounded bottom (Hellma Analytics, USA), and the sample was subjected to spectroscopic analysis. The sample was stirred using a magnetic stirrer rod. The difference between the three methods used is in the stirring setup. The fluorescence and CD spectrometers both have a built in stirrer system, for FCS the cuvette was kept on a magnetic stirrer plate. For FCS measurements, a $300 \mu 1$ aliquot was transferred to an 8-well chambered coverglass (Lab-Tek Chambered Coverglass, Thermo Scientific, USA). After FCS analysis, the aliquot was returned to the bulk and stirred until the time for the next recording.

The quantum dots were suspended in water and sonicated for 30 min prior to the experiment. 
Fluorescence correlation spectroscopy FCS measurements were performed on a ConfoCor 2 system (Carl Zeiss, Jena, Germany), consisting of an inverted microscope equipped with a CApochromat $40 \times, \mathrm{NA}=1.2$, water immersion UV-VIS-IR objective. ThT fluorescence was excited using the $458 \mathrm{~nm}$ line of the Argon laser. The HFT 458 main dichroic beam splitter was used to separate the incident and emitted light. The pinhole size in front of the detector was $70 \mu \mathrm{m}$ (1 Airy). Laser intensity measured at the objective was $6 \mu \mathrm{W}$.

Standard solutions of Rhodamine 6G (Rh6G), 10-25 nM, were used for instrumental calibration. The same optical setting that was used for ThT was used for Rh6G. The diffusion time of Rh6G was determined to be $\tau_{\mathrm{D}, \mathrm{Rh} 6 \mathrm{G}}=(24 \pm 2) \mu \mathrm{s}(\mathrm{n}=460)$, the counts per molecule and second $(\mathrm{CPMS})$ was $\mathrm{CPMS}=(5.1 \pm 0.6) \mathrm{kHz}$, and the structure parameter was $S^{2}=7 \pm$ 1.

Fluorescence intensity fluctuations were analyzed using temporal autocorrelation analysis. The thus generated autocorrelation curves (ACCs) were fitted with a model for free 3D diffusion of one component (23-25):

$$
\mathrm{G}(\tau)=\frac{1}{\mathrm{~N}} *\left(\frac{1}{\left(1+\frac{\tau}{\tau_{\mathrm{D}}}\right) * \sqrt{1+\frac{\tau}{S^{2} * \tau_{\mathrm{D}}}}}\right)+1
$$

where $\mathrm{N}$ is the average number of aggregates in the detection volume, $\tau_{\mathrm{D}}$ is the diffusion time, $S$ is the structure parameter.

Fluorescence spectroscopy A Jobin Yvon Horiba Fluorolog 3 (Longjumeau, France) unit was used to follow the increase in ThT fluorescence at $480 \mathrm{~nm}$ (excitation $440 \mathrm{~nm}$ ) upon aggregation of $10 \mu \mathrm{M}$ A $\beta 42$ peptide in $20 \mathrm{mM}$ HEPES, pH 7.0 at $20{ }^{\circ} \mathrm{C}$. The excitation and emission slits were set at $4 \mathrm{~nm}$. 
Circular dichroism spectroscopy CD spectra were measured on a Chirascan spectrometer (Applied Photophysics) equipped with a Peltier temperature control system. Measurements were performed at $20{ }^{\circ} \mathrm{C}$ using a cell with $4 \mathrm{~mm}$ path length with continuous mixing. The spectral region was recorded from 240 to $190 \mathrm{~nm}$. The samples contained $10 \mu \mathrm{M} \mathrm{A} \beta 42$ peptide at $\mathrm{pH} 7.0$.

Aggregation parameters were determined by fitting the time dependence of the CD signal intensity at $195 \mathrm{~nm}$, the ThT emmission at $480 \mathrm{~nm}$, and the distribution of diffusion times to equation:

$$
X(t)=X_{0}+\frac{A}{1+e^{-k\left(t-t_{\text {half }}\right)}}
$$

Where $\mathrm{X}(\mathrm{t})$ is in the case of fluorescence spectroscopy bulk florescence emission at $480 \mathrm{~nm}$ at time $t$, in the case of $\mathrm{CD}$ it is the signal intensity at $195 \mathrm{~nm}$ at time $t$, and in the case of FCS the diffusion time at time $t, \mathrm{X}_{0}$ is the value of the corresponding entity in the beginning of the aggregation process, at $t=0 \mathrm{~min}, \mathrm{~A}$ is the amplitude, $k$ the kinetic rate constant and $t_{\text {half }}$ the time when the signal intensity has reached half of its maximum value, that is the time that is required for the reaction to reach half-completion.

\section{Analysis of autocorrelation curves}

Using the Maximum Entropy Method for FCS (MEMFCS) [1] for systematic analysis of the recorded ACCs, we observed that most ACCs at early reaction time points (Fig 1A-C) can be fitted using a model for free 3D diffusion of a single component (Fig. S1). However, autocorrelation curves with a steeper slope were recorded towards the end of the aggregation process, which could be better fitted using an exponential decay model (Fig 1D-E). This change in shape may be an artifact arising due to short measurement times, as we discuss in 
detail in the subsection below (which we have excluded by control experiments (Figs S2S7)), but it may also reflect some real processes: (1) ThT binding/unbinding - as A $\beta$ aggregates grow, their diffusion becomes slower and the diffusion time may become longer than the chemical relaxation time of ThT binding/unbinding; (2) contribution from directed flow due to sedimentation - as the aggregates grow they start to sediment, giving rise to directed motion in addition to free 3D diffusion; (3) three-dimensional Brownian diffusion of rod-like macromolecules in the presence of randomly distributed spherical obstacles - as A $\beta$ aggregation proceeds, aggregates of various shapes and sizes form and their transporting properties are no longer well represented by the simple model for free $3 \mathrm{D}$ diffusion of small spheres [2].

(1) For short reaction times, $t<20 \mathrm{~min}$, small $\mathrm{A} \beta$ aggregates, $\tau_{\mathrm{D}}<1 \mathrm{~ms}$, are typically observed and the characteristic time of the ThT binding/unbinding reaction is longer than the characteristic time for diffusion. Under these conditions ThT molecules are either free or bound to $A \beta$ aggregates as they diffuse through the OVE, but they rarely dissociate during this time. Hence, only diffusion of ThT-labeled aggregates is observed since unbound ThT is not fluorescent. As the aggregates grow and their diffusion becomes slower, $\tau_{\mathrm{D}}>5 \mathrm{~ms}$, ThT molecules may pass between free and bound states while diffusing through the OVE. Hence, an ACC curve with two characteristic decay times may be observed, one reflecting the chemical relaxation of ThT binding/unbinding, estimated to be $\tau_{\text {chem }} \sim 5 \mathrm{~ms}$, and the other reflecting the diffusion of large $A \beta$ aggregates [3]. The chemical relaxation time estimated by FCS to be $\sim 5 \mathrm{~ms}$, agrees with the value previously measured by stopped-flow [4].

(2) and (3) Aggregate growth and the onset of sedimentation, which inevitably occurs as the size of amyloid aggregates increases [5,6], may give rise to directed flow and also contribute 
to the altered shape of ACCs that was observed in some ACCs recorded towards the end of the aggregation process.

We could not efficiently distinguish between the contribution of ThT binding/unbinding and directed flow due to sedimentation to the change in ACCs shape. However, irrespective of which method was used to determine the decay time of the ACCs - either by estimating the width at half-maximum or by fitting the ACCs using autocorrelation functions for free 3D diffusion or autocorrelation function for exponential decay with free 3D diffusion, the effect on the overall distribution of characteristic decay times was minimal and could be neglected (Fig. S7). Hence, the present use of multiple short signal acquisition times and a fitting model with lower number of free parameters makes the evaluation of ACCs more straightforward than in the case of longer signal acquisition times - since the system is dynamic and changes over time, complex ACCs with more than one characteristic decay time are obtained when longer measurement times are used. This necessitates ACCs evaluation by more complicated fitting functions, i.e. the use of multi-component models with several free fit parameters [79]. However, the use of multi-component models with several free fit parameters for fitting ACCs is not bias free, as it will allow for a perfect fit even if a wrong model function is used.

\section{Effect of FCS measurement time on autocorrelation curves}

In FCS, fluorescence intensity fluctuations are recorded over a certain period of time and statistical methods of analysis are applied to detect non-randomness in the data. Typically, this is done by temporal autocorrelation analysis, which was also used here to derive $\tau_{\mathrm{D}}$ and $\mathrm{N}$. The longer the measurement time, the better is the statistical analysis of underlying fluctuations and the resulting ACC is less noisy. When FCS is used to study a system that is in equilibrium or in a steady state, i.e. a system whose macroscopic properties are not changing over time, long measurement times can be used. However, when a dynamic process 
is being studied, properties of the investigated system are changing over time and the FCS measurement time needs to be adjusted to the time-course of the process that is being investigated. Since accurate FCS measurements require statistical analysis of many fluctuations, it is very important to know the limit to which the measurement time can be shortened without producing systematic errors in the estimation of molecular numbers and diffusion times [1,10-12]. The shortest characteristic decay time measured in our studies was $200 \mu \mathrm{s}$ and the longest one analyzed by fitting was $8 \mathrm{~ms}$. For aggregates characterized by $\tau_{\mathrm{D}}$ $=200 \mu \mathrm{s}$, the average number of passages across the OVE observed during a $10 \mathrm{~s}$ measurement is $5 \times 10^{4}$, and for the largest aggregates analyzed, $\tau_{\mathrm{D}}=8 \mathrm{~ms}$, the average number of passages across the OVE are observed during a $10 \mathrm{~s}$ measurement is 1250 . A $10 \mathrm{~s}$ measurement time is therefore more than 1000 times longer than the characteristic time of the slowest process analyzed. Under such conditions, the standard deviation (SD) in $\mathrm{N}$ and $\tau_{\mathrm{D}}$ arising due to particle noise should be SD $<25 \%[13,14]$. In order to verify that this is also true for our measurements, the distribution of diffusion times was determined for Rh6G in water, $\tau_{\mathrm{D}, \mathrm{Rh} 6 \mathrm{G}}=(24 \pm 2) \mu \mathrm{s}$ (Fig. S2); sonicated quantum dots in aqueous suspension, $\tau_{\mathrm{D}, \mathrm{QD}}$ in water $=(273 \pm 11) \mu \mathrm{s}\left(\right.$ Fig. S3); and quantum dots suspension in $40 \%$ sucrose, $\tau_{\mathrm{D}, \mathrm{QD} \text { in sucrose } 40}=$ $(748 \pm 29) \mu$ s (Fig. S4), in $60 \%$ sucrose, $\tau_{\mathrm{D}, \mathrm{QD}}$ in sucrose60 $=(3643 \pm 303) \mu \mathrm{s}$ (Fig. S5), and in 70 $\%$ sucrose, $\tau_{\mathrm{D}, \mathrm{QD}}$ in sucrose70 $=(9665 \pm 799) \mu$ s $($ Fig. S6), using the same optical setting as for ThT-A A $\beta$ aggregation. In all cases the $\mathrm{SD}<10 \%$ and significantly smaller than the spreading of diffusion times measured during $A \beta$ aggregation (Figs 1, 2 and 4, S7). Notwithstanding, for very large aggregates, $\tau_{\mathrm{D}}>50 \mathrm{~ms}$, the number of passages becomes too low and the statistical analysis becomes unreliable. Such large aggregates where therefore not considered. 


\section{Effect of brightness on FCS analysis}

Since the analyzed system is heterogeneous, containing different amounts of aggregates of different sizes and brightness, we typically observe overlapping ACCs (Fig. 1A and B, red, yellow, green, blue, magenta, dark ochre and black), which reflect phenomenological properties of the most abundant entity. But we also occasionally observe ACCs that markedly differ in amplitude and characteristic decay times from the typically observed ones, reflecting sporadic observation of larger and brighter aggregates (Fig. 1 A and B, light blue, ochre, cyan). At the beginning of the aggregation process, just after $A \beta$ solubilization $(t=0 \mathrm{~min})$, the number of such events is typically low -2 to 3 ACCs out of 30 show amplitudes and/or characteristic decay times that markedly differ from the corresponding values determined in the remaining measurements (Fig. 1C). During the course of aggregation, the number of larger (Fig. 2A) and brighter (Fig. 2C) aggregates progressively increases, and longer characteristic decay times are recorded at later stages of the aggregation process (Fig. 1C and F, Fig. 2B and C and Fig. 4).

The brightness distribution that builds up during the aggregation process (Fig. 2C) may affect correct determination of particle numbers - when two species of different brightness coexist the apparent number of molecules in the OVE determined from the amplitude of the ACC will be lower than the total number of molecules but higher than the number of brighter molecules $[13,14]$. However, this difference is not large when the molecules are of similar brightness. For example, in a mixture that contains equal amounts of molecules that differ in brightness by a factor of two, there appears to be $10 \%$ less molecules overall $[13,14]$. Hence, small uncertainties in the apparent number of molecules in the lag phase can be expected. As the aggregation process proceeds, the difference in brightness between small and large aggregates increases several times (Fig. 2C). Under such conditions, when large differences in brightness exist, FCS analysis becomes "skewed" towards reflecting dynamical properties 
of the brightest entities. Hence, individual ACCs exhibit one principal characteristic decay time and can be effectively evaluated using a single diffusion component model (Eq. 1), which yields one amplitude and one characteristic decay time that reflect macroscopic properties of the brightest aggregate (Fig. 1 D-F, Fig. S8). Unfortunately, due to this, the information on the concentration of the dimmer small aggregates may get lost and their concentration may become underestimated at later stages of the aggregation process.

\section{References}

1. Sengupta P, Garai K, Balaji J, Periasamy N, Maiti S (2002) Measuring size distribution in highly heterogeneous systems with fluorescence correlation spectroscopy. Biophys $J$ 84(3): 1977-1984.

2. Fazl H and Sakha F (2010) Three-dimensional Brownian diffusion of rod-like macromolecules in the presence of randomly distributed spherical obstacles: Molecular dynamics simulation. J. Chem. Phys. 133(23): 234904:1-6.

3. Elson EL (2001) Fluorescence correlation spectroscopy measures molecular transport in cells. Traffic 2(11): 789-796.

4. LeVine H (1997) Stopped-flow kinetics reveal multiple phases of thioflavin T binding to alzheimer beta(1-40) amyloid fibrils. Arch Biochem Biophys 342(2): 306-316.

5. Dam J, Schuck P (2004) Calculating sedimentation coefficient distributions by direct modeling of sedimentation velocity concentration profiles. Methods Enzymol 384: 185212.

6. van Oss CJ (2006) in Interfacial Forces in Aqueous Media, Second Edition, (CRC Press, pp 75-77.

7. Mittag JJ, Milani S, Walsh DM, Radler JO, McManus JJ (2014) Simultaneous measurement of a range of particle sizes during Abeta1-42 fibrillogenesis quantified using fluorescence correlation spectroscopy. Biochem Biophys Res Commun 448(2): 195-199.

8. Matsumura S, et al. (2011) Two distinct amyloid beta-protein (A beta) assembly pathways leading to oligomers and fibrils identified by combined fluorescence correlation spectroscopy, morphology, and toxicity analyses. J Biol Chem 286(13): $11555-11562$.

9. Ries J,Schwille P (2008) New concepts for fluorescence correlation spectroscopy on membranes. Phys Chem Chem Phys 10(24): 3487-3497.

10. Qian H, Elson EL,Frieden C (1992) Studies on the structure of actin gels using time correlation spectroscopy of fluorescent beads. Biophys J 63(4): 1000-1010.

11. Saffarian S,Elson EL (2003) Statistical analysis of fluorescence correlation spectroscopy: The standard deviation and bias. Biophys $J$ 84(3): 2030-2042.

12. Wohland T, Rigler R,Vogel H (2001) The standard deviation in fluorescence correlation spectroscopy. Biophys J 80(6): 2987-2999.

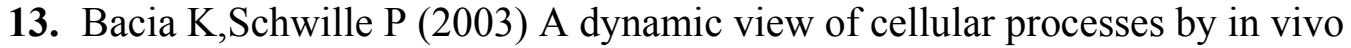
fluorescence auto- and cross-correlation spectroscopy. Methods 29(1): 74-85.

14. Ries J, et al (2010) Automated suppression of sample-related artifacts in fluorescence correlation spectroscopy. Opt Express 18(11): 11073-11082. 
Table S1

\begin{tabular}{|l|c|c|}
\hline & $\boldsymbol{k}\left(\mathbf{m i n}^{-1}\right)$ & $\boldsymbol{t}_{\text {half }}$ (min) \\
\hline All data (n=3) & $0.08 \pm 0.05$ & $24 \pm 7$ \\
\hline Five highest (n=3) & $0.12 \pm 0.04$ & $28 \pm 8$ \\
\hline Five lowest (n=3) & $0.14 \pm 0.05$ & $23 \pm 12$ \\
\hline
\end{tabular}

Table S1. Kinetic parameters obtained from FCS measurements in a solution of $10 \mu \mathrm{M}$ A $\beta 42$ in $20 \mathrm{mM}$ HEPES, pH 7.0 and $10 \mu \mathrm{M}$ ThT at $20{ }^{\circ} \mathrm{C}$ by analyzing the data with longest decay times (5), shortest decay times (5) and all other data (20). Differences were not observed. 
Figure S1
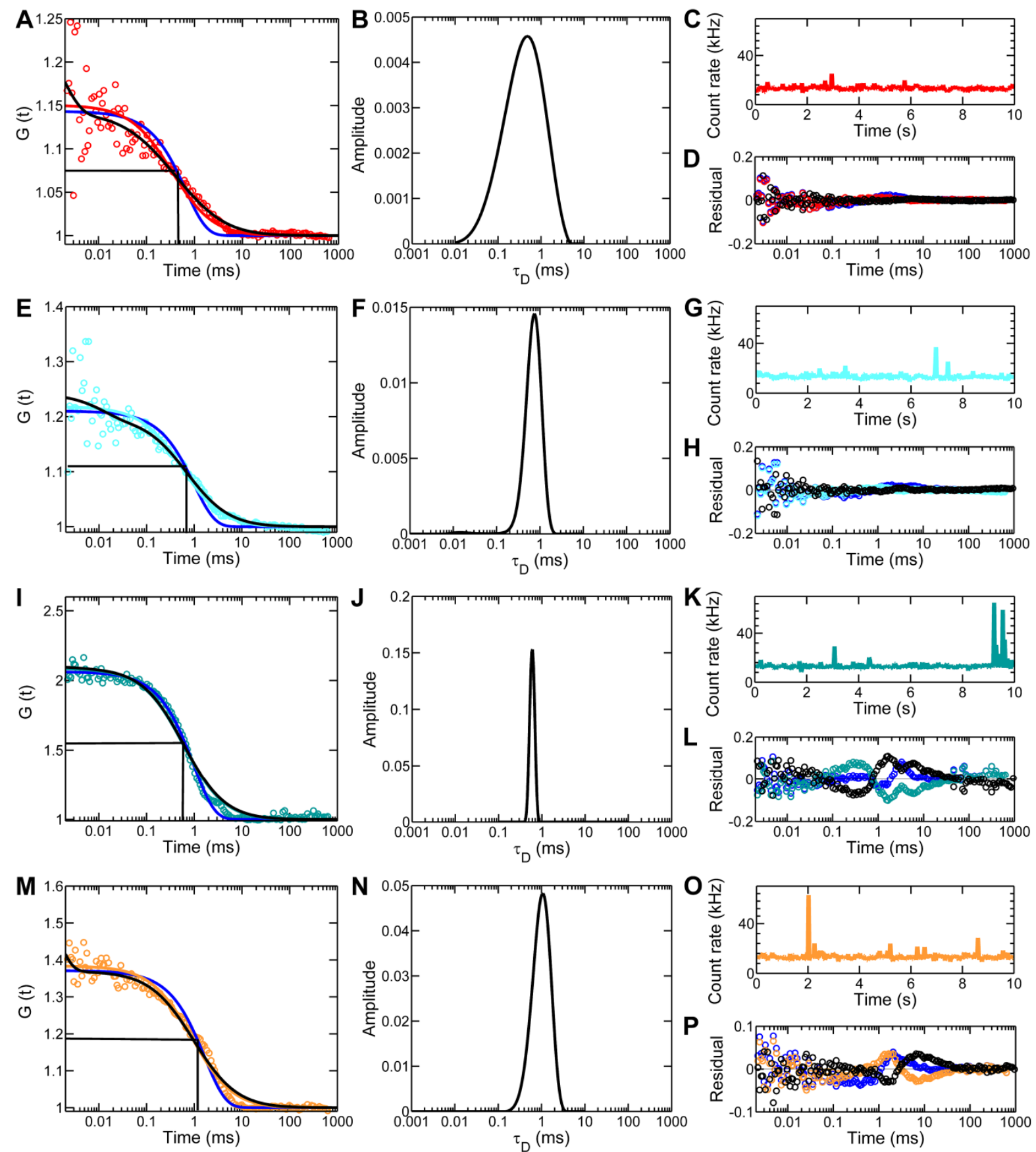

Figure S1. Analysis of ACCs recorded in a solution of $10 \mu \mathrm{M} \mathrm{A} \beta 42$ and $10 \mu \mathrm{M}$ ThT in 20 $\mathrm{mM}$ HEPES, $\mathrm{pH} 7.0, \mathrm{~T}=20{ }^{\circ} \mathrm{C}$ at $t=0 \mathrm{~min}$ using different methods. A, E, I and $\mathbf{M}$. Experimentally derived ACCs (open circles) evaluated using the model for free 3D diffusion (Eq. 1, correspondingly colored line), free 3D diffusion with kinetics (blue line), the MEMFCS method (black line) and the width at half-maximum (vertical black line). B, F, J and $\mathbf{N}$. Distribution of diffusion times as determined by MEMFCS. $\mathbf{C}, \mathbf{G}, \mathbf{K}$ and $\mathbf{O}$. Fluorescence intensity fluctuation traces, with each individual measurement lasting $10 \mathrm{~s}$. D, H, L and P. Residuals for different fitting methods: Eq. 1 (corresponding color), free 3D diffusion with kinetics (blue) and MEMFCS (black). 
Figure S2
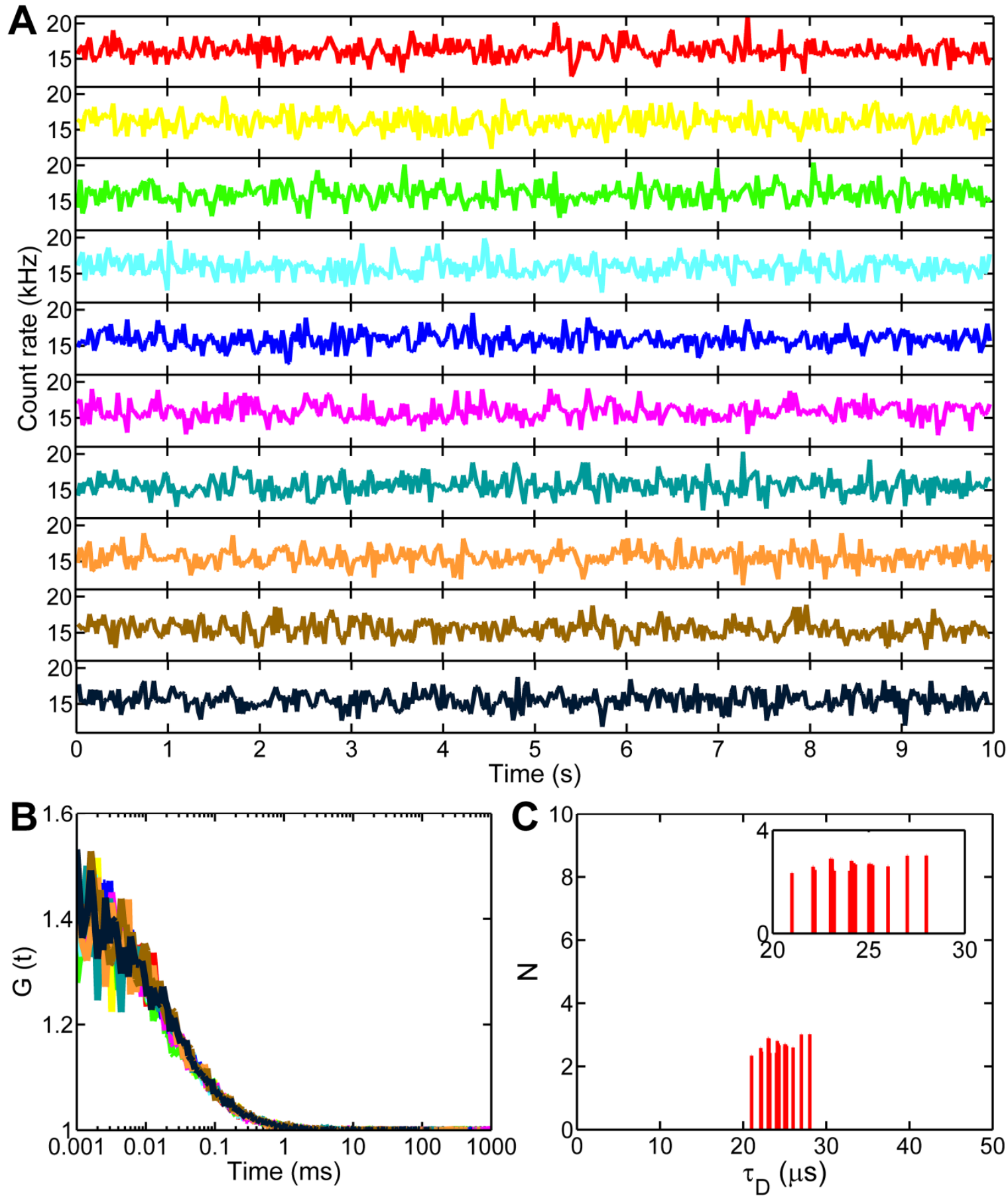

Figure S2. FCS measurements in a $25 \mathrm{nM}$ standard solution of Rh6G in water. A. Ten consecutively recorded fluorescence intensity fluctuation traces, with each individual measurement lasting $10 \mathrm{~s}$. B. Corresponding autocorrelation curves. C. Diffusion time distribution histogram generated by plotting the diffusion time $\left(\tau_{\mathrm{D}}\right)$ as a function of the apparent number of Rh6G molecules $(\mathrm{N})$ obtained by fitting 30 consecutively acquired ACCs with Eq 1. On panels A and B 10 consecutive measurements, out of 30, are shown for clarity. The fluorescence intensity time series and the corresponding ACC are shown in the same color. $\mathrm{N}=2.6 \pm 0.3 ; \tau_{\mathrm{D}, \mathrm{Rh} 6 \mathrm{G}}=(24 \pm 2) \mu \mathrm{s} ; \mathrm{CPMS}=(5.1 \pm 0.6) \mathrm{kHz} ; \mathrm{n}=30$. 
Figure S3
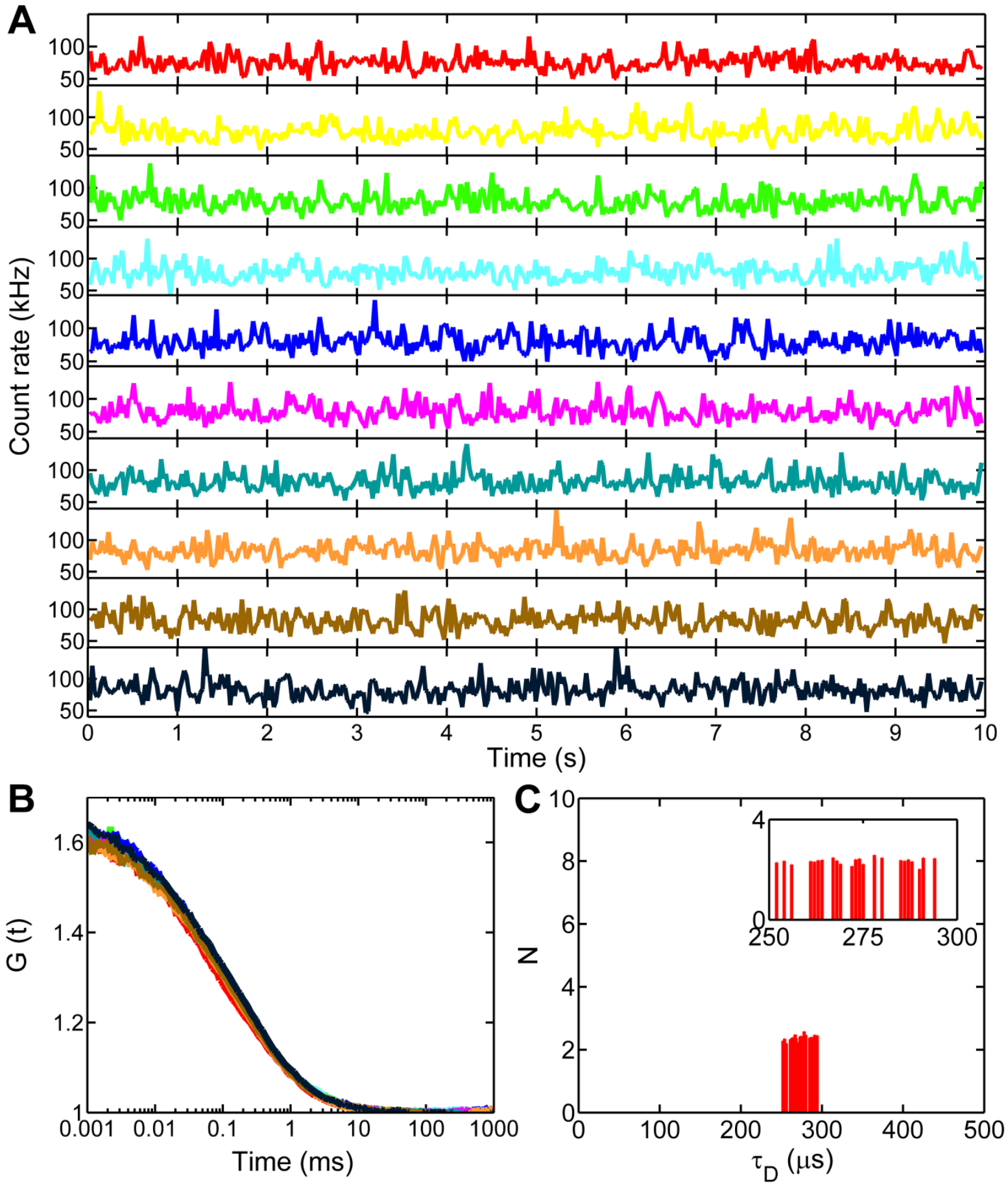

Figure S3. FCS measurements in a standard solution of quantum dots in water. A. Ten consecutively recorded fluorescence intensity fluctuation traces, with each individual measurement lasting $10 \mathrm{~s}$. B. Corresponding autocorrelation curves. C. Diffusion time distribution histogram generated by plotting the diffusion time $\left(\tau_{\mathrm{D}}\right)$ as a function of the apparent number of quantum dots $(\mathrm{N})$ obtained by fitting 30 consecutively acquired ACCs with Eq 1. On panels A and B 10 consecutive measurements, out of 30, are shown for clarity. The fluorescence intensity time series and the corresponding ACC are shown in the same color. $\mathrm{N}=2.3 \pm 0.1 ; \tau_{\mathrm{D}}=(270 \pm 10) \mu \mathrm{s} ; \mathrm{CPMS}=(50 \pm 3) \mathrm{kHz} ; \mathrm{n}=30$. 
Figure S4
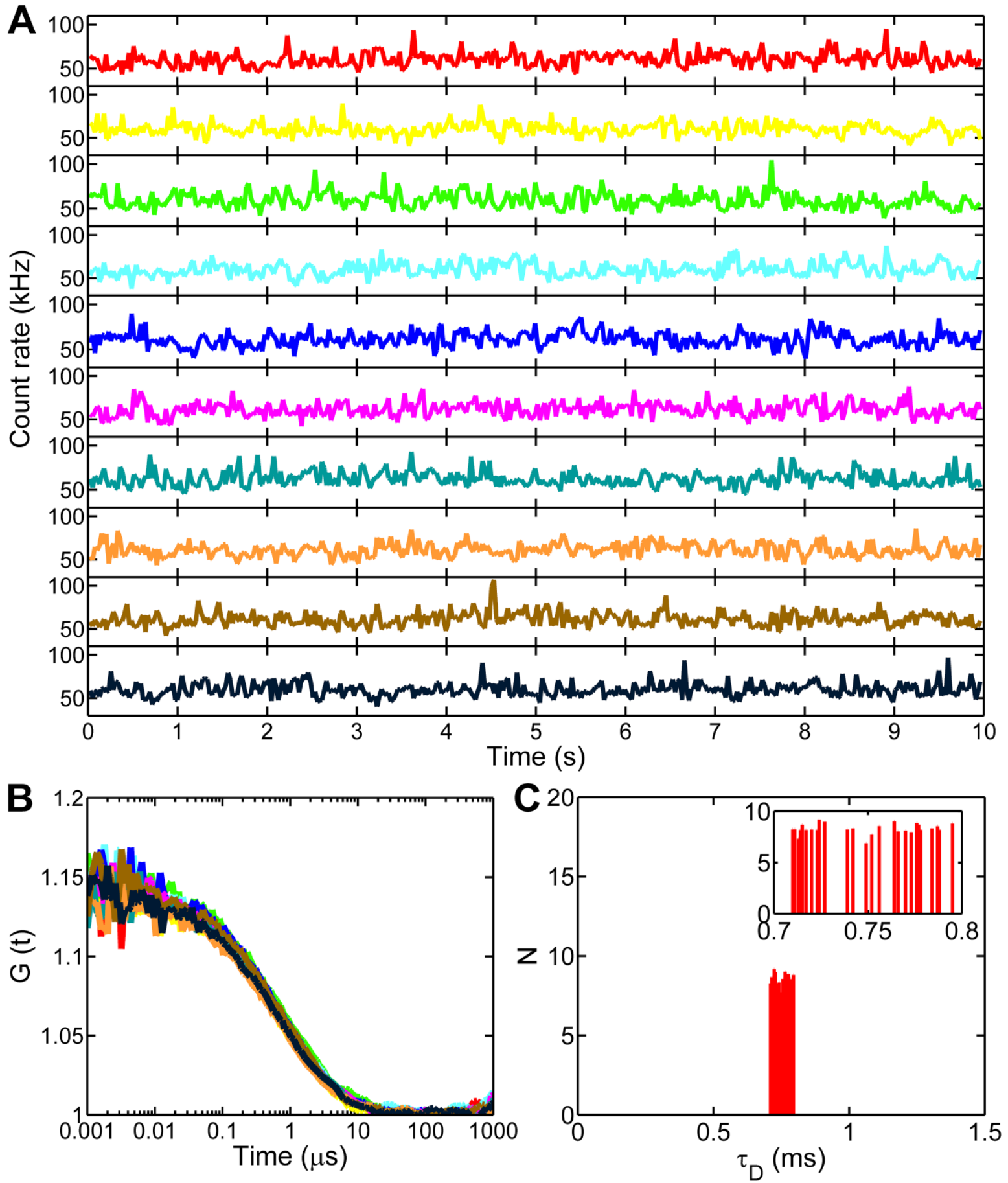

Figure S4. FCS measurements in a standard solution of quantum dots in $40 \%$ sucrose. A. Ten consecutively recorded fluorescence intensity fluctuation traces, with each individual measurement lasting $10 \mathrm{~s}$. B. Corresponding autocorrelation curves. C. Diffusion time distribution histogram generated by plotting the diffusion time $\left(\tau_{\mathrm{D}}\right)$ as a function of the apparent number of quantum dots $(\mathrm{N})$ obtained by fitting 30 consecutively acquired ACCs with Eq 1. On panels A and B 10 consecutive measurements, out of 30, are shown for clarity. The fluorescence intensity time series and the corresponding ACC are shown in the same color. $\mathrm{N}=8.2 \pm 0.5 ; \tau_{\mathrm{D}}=(750 \pm 30) \mu \mathrm{s} ; \mathrm{CPMS}=(8.4 \pm 0.5) \mathrm{kHz} ; \mathrm{n}=30$. 
Figure S5
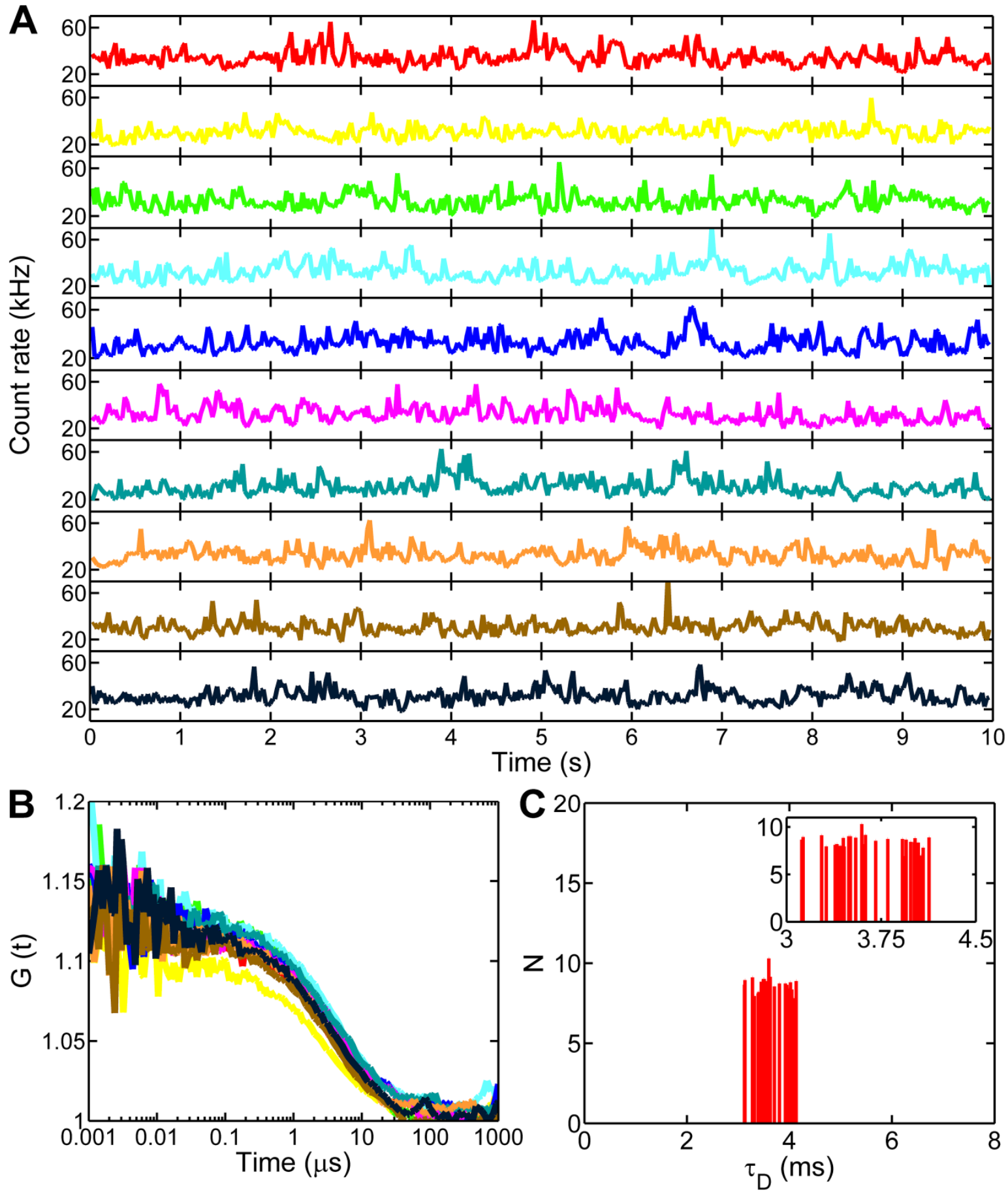

Figure S5. FCS measurements in a standard solution of quantum dots in $60 \%$ sucrose. A. Ten consecutively recorded fluorescence intensity fluctuation traces, with each individual measurement lasting $10 \mathrm{~s}$. B. Corresponding autocorrelation curves. C. Diffusion time distribution histogram generated by plotting the diffusion time $\left(\tau_{\mathrm{D}}\right)$ as a function of the apparent number of quantum dots $(\mathrm{N})$ obtained by fitting 30 consecutively acquired ACCs with Eq 1. On panels A and B 10 consecutive measurements, out of 30, are shown for clarity. The fluorescence intensity time series and the corresponding ACC are shown in the same color. $\mathrm{N}=8.4 \pm 0.6 ; \tau_{\mathrm{D}}=(3600 \pm 300) \mu \mathrm{s} ; \mathrm{CPMS}=(4.2 \pm 0.4) \mathrm{kHz} ; \mathrm{n}=30$. 
Figure S6
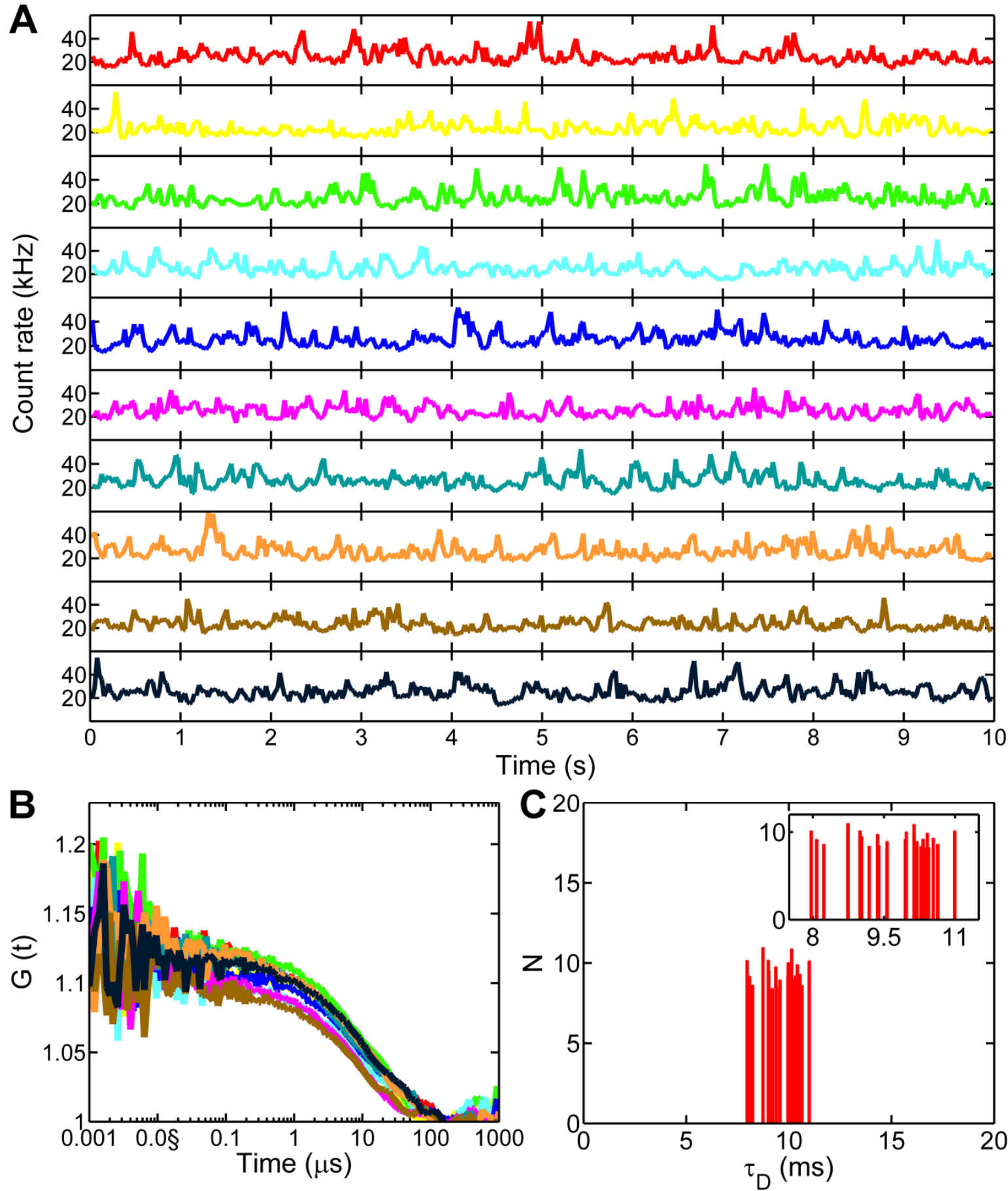

Figure S6. FCS measurements in a standard solution of quantum dots in $70 \%$ sucrose. A. Ten consecutively recorded fluorescence intensity fluctuation traces, with each individual measurement lasting $10 \mathrm{~s}$. B. Corresponding autocorrelation curves. C. Diffusion time distribution histogram generated by plotting the diffusion time $\left(\tau_{\mathrm{D}}\right)$ as a function of the apparent number of quantum dots $(\mathrm{N})$ obtained by fitting 30 consecutively acquired ACCs with Eq 1. On panels A and B 10 consecutive measurements, out of 30, are shown for clarity. The fluorescence intensity time series and the corresponding ACC are shown in the same color. $\mathrm{N}=9.1 \pm 0.8 ; \tau_{\mathrm{D}}=(9600 \pm 800) \mu \mathrm{s} ; \mathrm{CPMS}=(3.3 \pm 0.4) \mathrm{kHz} ; S^{2}=7.0 \pm 1 ; \mathrm{n}=30$. 


\section{Figure S7}
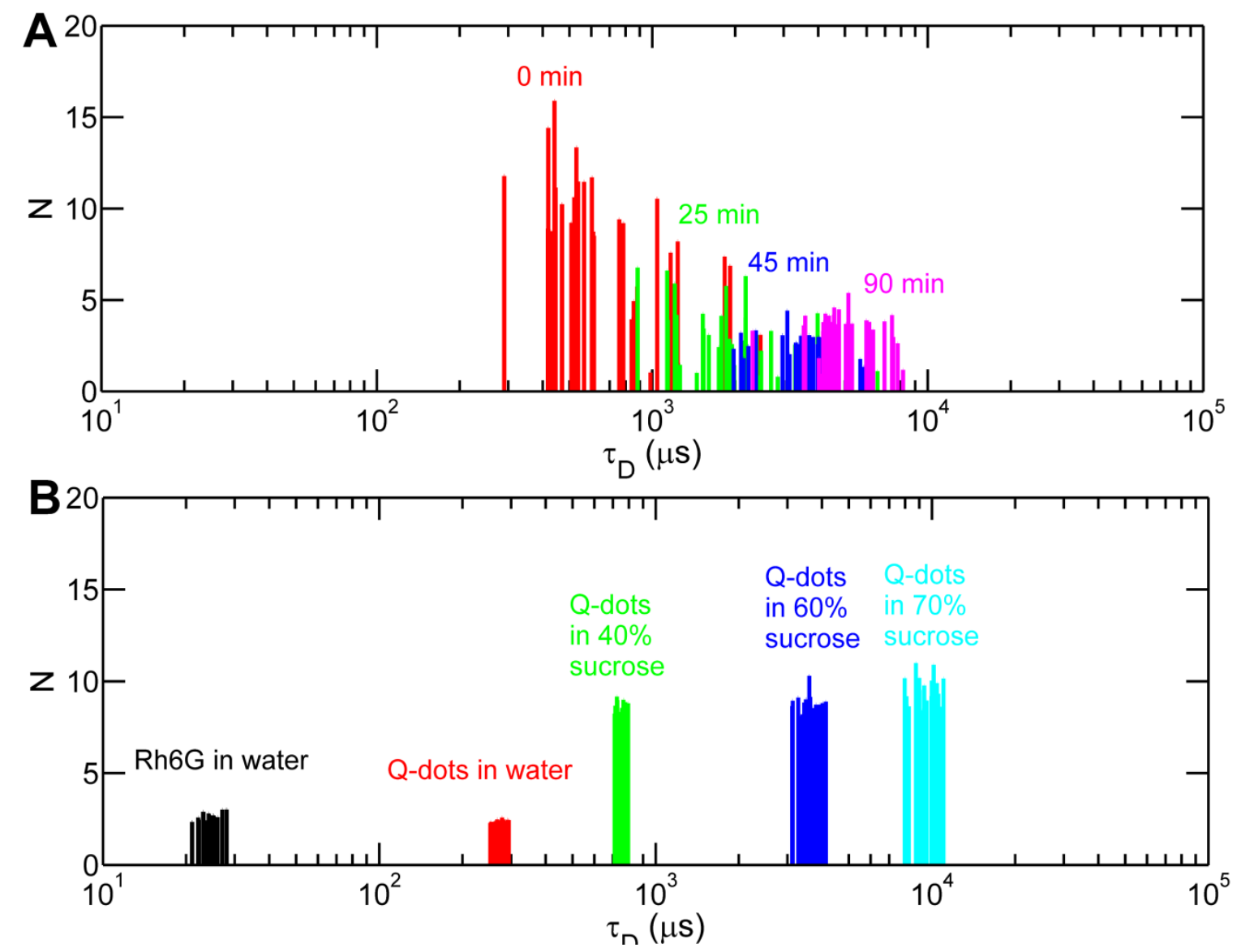

Figure S7. Distribution of diffusion times during $A \beta 42$ aggregation is much broader than the distribution of diffusion times in homogeneous standard solution samples with matching diffusion times. A. Distribution of diffusion times at different time points during A $\beta 42$ aggregation in a solution of $10 \mu \mathrm{M} \mathrm{A} \beta 42$ and $10 \mu \mathrm{M}$ ThT in $20 \mathrm{mM}$ HEPES, pH 7.0, T = 20 ${ }^{\circ} \mathrm{C}$ : at the beginning, $t=0 \mathrm{~min}$ (red); at $t=25 \mathrm{~min}$ (green); at $t=45 \mathrm{~min}$ (blue); at $t=90 \mathrm{~min}$ (magenta). B. Distribution of diffusion times in standard solutions of Rhodamine 6G in water (black); quantum dots in water (red); quantum dots in $40 \%$ sucrose (green); quantum dots in $60 \%$ sucrose (blue); quantum dots in $70 \%$ sucrose (cyan). Control samples were selected to span the whole region of diffusion times observed during $A \beta 42$ aggregation. 
Figure S8
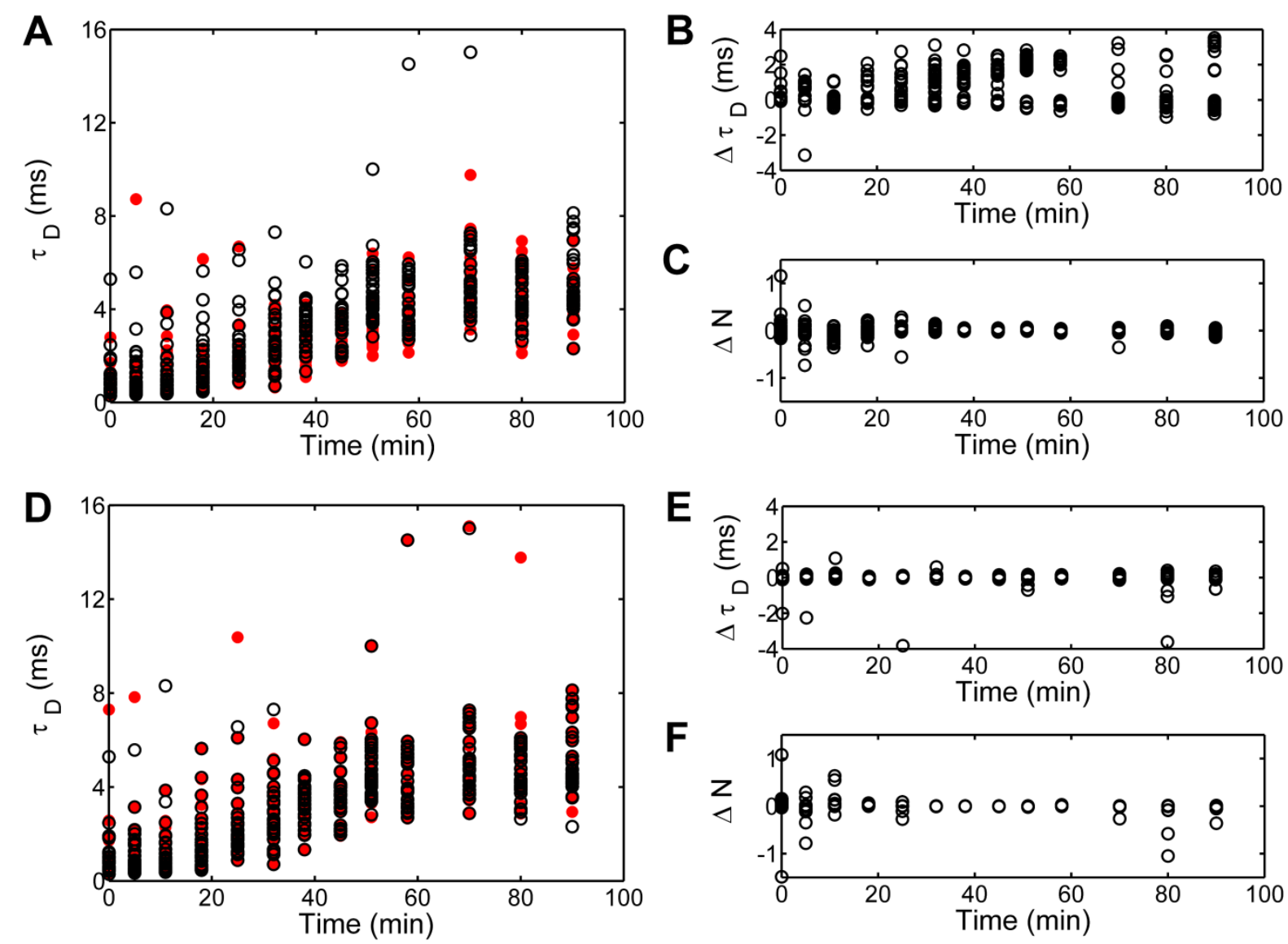

$\mathbf{E}$

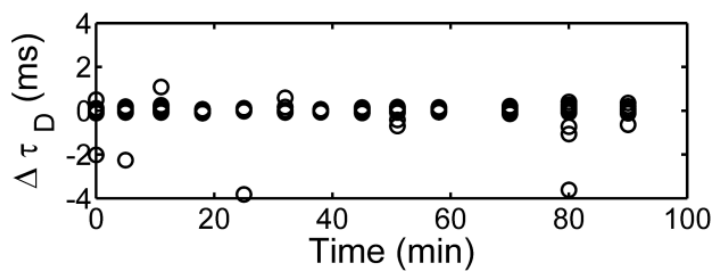

$\mathbf{F}$
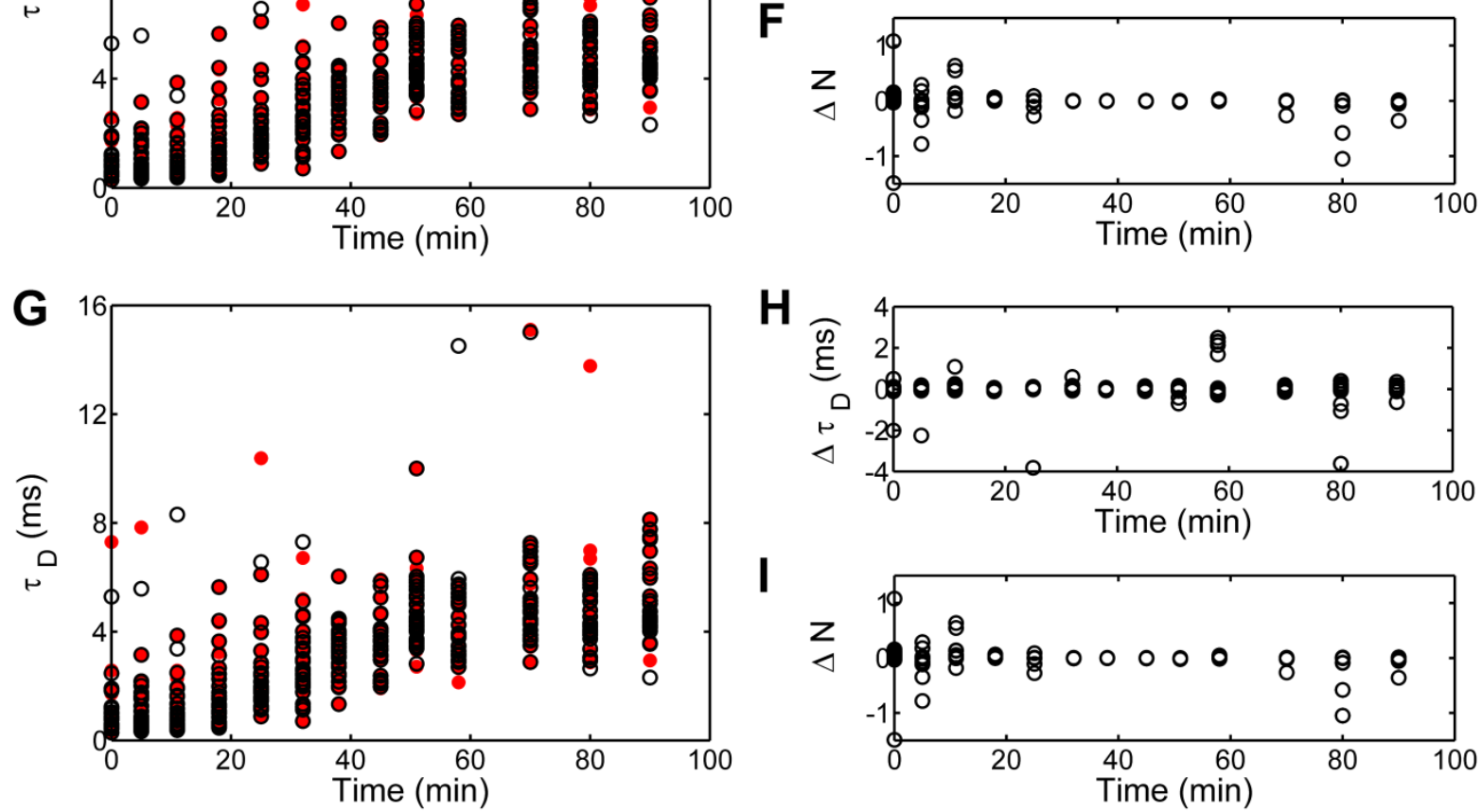

I

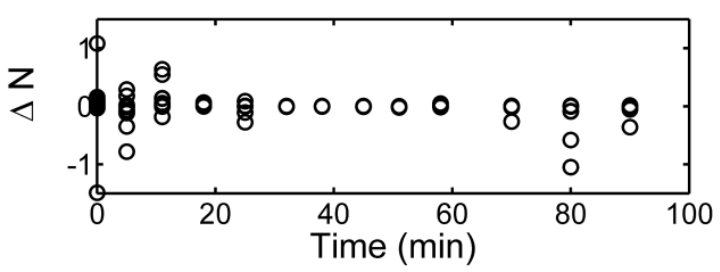

Figure S8. Effect of different ACC evaluation methods on the overall distribution of diffusion times measured in a solution of $10 \mu \mathrm{M}$ A $\beta 42$ and $10 \mu \mathrm{M}$ ThT in $20 \mathrm{mM}$ HEPES, $\mathrm{pH}$ 7.0, $\mathrm{T}=20{ }^{\circ} \mathrm{C}$ during the aggregation process. A.-C. A. Differences in diffusion time distribution during A $\beta 42$ aggregation obtained using the model for free 3D diffusion with a kinetic part (Eq. 1a) when all fitting parameters are free (open circles) and the structure parameter is fixed $S^{2}=7.0$ (red dots). B. Effect on the diffusion time. C. Effect on the number of molecules. D.-F. D. Differences in diffusion time distribution during A $\beta 42$ aggregation obtained using the model for free 3D diffusion with a kinetic part (Eq. 1a) when all fitting parameters are free (open circles) and the model for free 3D diffusion (Eq. 1) when all fitting parameters are free (red dots). E. Effect on the diffusion time. F. Effect on the number of molecules. G.-I. G. Differences in diffusion time distribution during A $\beta 42$ 
aggregation obtained using the model for free 3D diffusion with a kinetic part (Eq. 1a) when all fitting parameters are free (open circles) and the model for free 3D diffusion (Eq. 1) when the structure parameter is fixed $S^{2}=7.0$ (red dots). H. Effect on the diffusion time. I. Effect on the number of molecules.

$$
\begin{aligned}
& G(x)=\frac{1}{N} *\left(1+\frac{A}{1-A} * e^{-\frac{x}{t_{C}}}\right) *\left(\frac{1}{\left(1+\frac{x}{\tau_{D}}\right) * \sqrt{1+\frac{x}{S^{2}{ }^{*} \tau_{D}}}}\right)+1 \\
& \mathrm{G}(\tau)=\frac{1}{\mathrm{~N}} *\left(\frac{1}{\left(1+\frac{\tau}{\tau_{\mathrm{D}}}\right) * \sqrt{1+\frac{\tau}{S^{2} * \tau_{\mathrm{D}}}}}\right)+1
\end{aligned}
$$

where $N$ is the average number of particles in the OVE, $A$ is dark state fraction, $t_{c}$ is chemical relaxation time constant, $\mathrm{t}_{\mathrm{D}}$ is the diffusion time, and $S^{2}$ is the structure parameter. 
Figure S9

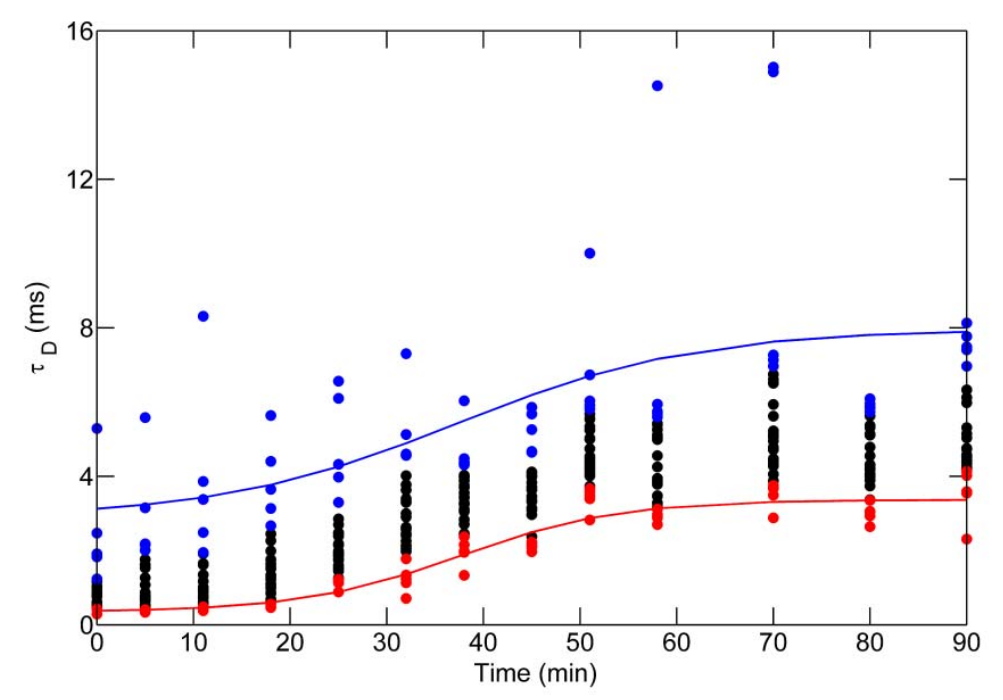

Figure S9. Fitting of the distribution of diffusion times measured by FCS in a solution of 10 $\mu \mathrm{M} A \beta 42$ and $10 \mu \mathrm{M}$ ThT in $20 \mathrm{mM}$ HEPES, pH 7.0 at $20{ }^{\circ} \mathrm{C}$, using only the five longest diffusion times (blue) or five shortest diffusion times (red) for each time point. Lines correspond to the fit of the diffusion times using Eq. 2. 
Figure S10
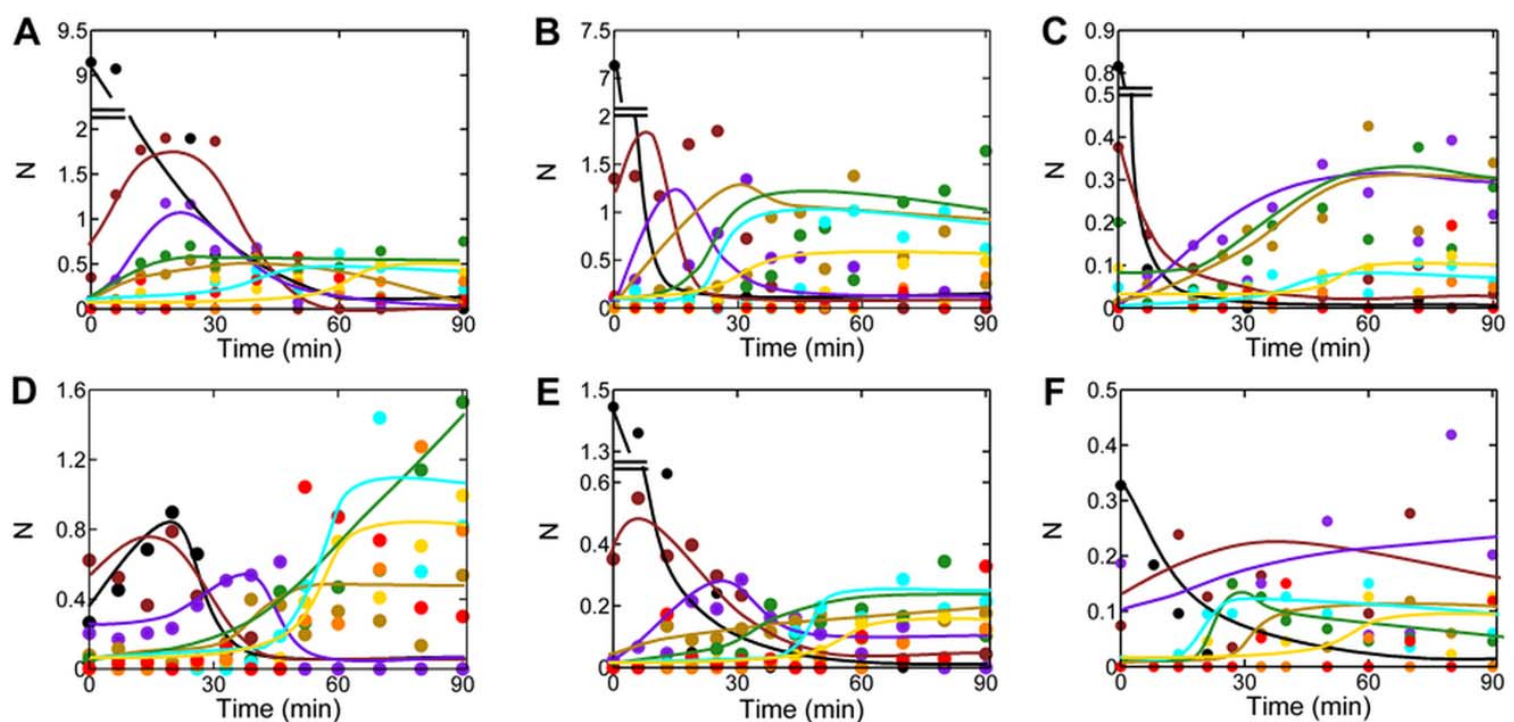

Figure S10. A.-C. Changes in the number of ThT-reactive aggregates with different diffusion times monitored using $10 \mu \mathrm{M} \mathrm{A} \beta$ and different ThT concentrations: (A) $20 \mu \mathrm{M}$, (B) $10 \mu \mathrm{M}$ and (C) $0.5 \mu \mathrm{M}$. D.-F. Changes in the number of ThT-reactive aggregates with different diffusion times monitored using $10 \mu \mathrm{M}$ ThT and different $\mathrm{A} \beta$ concentrations: (D) $20 \mu \mathrm{M}$, (E) $5 \mu \mathrm{M}$ and (F) $1.5 \mu \mathrm{M}$. On all figures: $\tau_{\mathrm{D}}<1 \mathrm{~ms}(\bullet), 1 \mathrm{~ms}<\tau_{\mathrm{D}}<2 \mathrm{~ms}(\bullet), 2 \mathrm{~ms}<\tau_{\mathrm{D}}<3 \mathrm{~ms}$ $(\bullet), 3 \mathrm{~ms}<\tau_{\mathrm{D}}<4 \mathrm{~ms} \mathrm{( \bullet ),} 4 \mathrm{~ms}<\tau_{\mathrm{D}}<5 \mathrm{~ms}(\bullet), 5 \mathrm{~ms}<\tau_{\mathrm{D}}<6 \mathrm{~ms} \mathrm{( \bullet ),} 6 \mathrm{~ms}<\tau_{\mathrm{D}}<7 \mathrm{~ms}$ $(\bullet), 7 \mathrm{~ms}<\tau_{\mathrm{D}}<8 \mathrm{~ms}(\bullet)$, and $\tau_{\mathrm{D}}>8 \mathrm{~ms}(\bullet)$. Lines were made by hand to help guide the eye. Experiments were carried out in $20 \mathrm{mM}$ HEPES, $\mathrm{pH} 7.0$, with continuous stirring at 20 ${ }^{\circ} \mathrm{C}$. 